Hartman, P. E., Hartman, Z. \& Serman, D. (1960). J.gen. Microbiol. 22, 354-368

\title{
Complementation Mapping by Abortive Transduction of Histidine requiring Salmonella Mutants
}

\author{
By P. E. HARTMAN, Z. HARTMAN AND D. SERMAN \\ Department of Biology, The Johns Hopkins University, Baltimore 18, \\ Maryland, U.S.A.
}

\begin{abstract}
SUMMARY: Determination has been made by the technique of abortive transduction of the functional relationships of over 200 Salmonella typhimurium histidine mutant alleles. The results are compared with those obtained by genetic recombination (Hartman, Loper \& Šerman, 1960) and enzyme studies (Ames, Garry \& Herzenberg, 1960). The results indicate that abortive transduction tests can be used to position rapidly sites of mutation on a chromosome map within the region of a single gene. The tests for genetic complementation reveal that some genes behave as single functional units, while other genes are composed of two, and some of four, functional subunits (complementation units). The existence of mutants with overlapping, intragenic non-complementing patterns may allow crude mapping, solely by complementation, of some mutational sites within certain single genes. In gene $D$, the ordering of mutational sites by this technique so far conforms with that obtained by the more classic methods of map construction based on inferences derived from recombination analyses. Data are presented bearing on the individuality of the gene and the effect of mutation thereon. It is concluded that some genetic damages, localized within single genes, can secondarily affect the expression of adjacent gene(s) in addition to their primary effect upon the gene in which they are located (position effect).
\end{abstract}

Stocker, Zinder \& Lederberg (1953) showed that genetic fragments exhibit two distinct types of behaviour following transduction into recipient bacteria. In 'complete' transduction, the genetic material is integrated into the replicating genome, resulting in true clonal inheritance of the transduced trait. In 'abortive' transduction, the transduced fragment is not integrated. Rather, the transduced genetic material is envisaged as existing in a non-replicating form, passing unilinearly from the recipient cell to only one of the daughter cells at each cell division (reviewed in Hartman, 1957; Hartman \& Goodgal, 1959). Thus at any given time there is present only one bacterium in the clone containing the genetic fragment (Stocker, 1956; Lederberg, 1956). The initial observations on transduction of genes engendering motility were extended to auxotrophic mutants by Ozeki (1956). Demerec and co-workers (e.g. Demerec \& Ozeki, 1959) have developed the abortive transduction test as a means of examining the functional relationships between genes of independently isolated mutants. The abortive transduction technique has provided a rapid test for functional allelism between phenotypically similar mutants (Demerec \& Ozeki, 1959; Demerec \& Hartman, 1959). In effect, a cell is constructed in which a specific small region of the chromosome may be examined for its behaviour in the heterozygous state. This allows determination of the recessive character of auxotrophic mutations in combination with wild-type alleles and the determination, in the critical trans configuration, of complementation 
between auxotrophic mutations. The current report describes complementation tests performed with histidine mutants of Salmonella typhimurium and compares these results with those obtained by other methods (Hartman, et al. 1960; Ames, et al. 1960).

\section{METHODS}

Many of the methods used in these studies and the genetic nomenclature used in this report are described in the preceding article (Hartman et al. 1960). Rapid screening of mutants to identify complementation patterns was achieved by diluting a saturated aerated nutrient broth culture 1/5 in minimal medium without carbon source and plating $0.1 \mathrm{ml}$. of the diluted suspension (about $5 \times 10^{7}$ bacteria plated) on minimal agar. As soon as the excess moisture had soaked into the medium, the plate was marked into 5 sectors and one drop of phage suspension $\left(1 \times 10^{9}\right.$ phage particles $/ \mathrm{ml}$.) spotted in each sector. The plates were then incubated at $37^{\circ}$ for 2 or 3 days before examination at magnification $\times 9$ to 20 by transmitted light under a dissecting microscope. Highly mutable strains may be examined in this manner as long as there are not present appreciable numbers of spontaneously formed very small colonies. The unspotted regions and a spot of phage previously grown on the recipient strain, or phage prepared on multisite mutations involving the loci under test, served as controls. In appropriate tests, the minute colonies were readily visible as long as the experiment was controlled with regard to the following respects. (1) Background growth of the plated strain must be held to a minimum during initial incubation of the plates, otherwise it interferes with the discernibility of the minute colonies as well as suppressing their growth. Thus minimal agar was vastly superior to EM medium. (2) Slightly dried plates afforded better visibility since the small clones tended under these conditions to form small piles rather than spreading over a larger area in a thin layer. (3) The large complete transductions must not be too numerous or they decrease the sizes of the minute colonies. (4) Similarly, an initial plating of more than about $5 \times 10^{7}$ bacteria suppressed minute colony growth. (5) Spotting of excessive concentrations of phage caused excessive lysis in the spotted areas; decreasing phage concentrations, below that specified, elicited fewer clones arising from abortive transduction. (5) Spotting of the phage suspensions must be performed shortly after the bacteria have been spread on the agar plates. (6) The bacteria must be distributed evenly and be spread to the edge of the plates in order to avoid excessive numbers of residual divisions by isolated single bacteria. Such microcolonies can, superficially, mimic the appearance of minute colonies formed following abortive transduction.

In further tests, $0.1 \mathrm{ml}$. of phage suspension (usually about $5 \times 10^{8}$ particles) was added to $0.9 \mathrm{ml}$. diluted bacterial suspension, absorbed $5 \mathrm{~min}$. at $37^{\circ}$, and $0.1 \mathrm{ml}$. spread on minimal agar plates. After 2 or 3 days of incubation at $37^{\circ}$ the large colonies, arising as a result of complete transduction, were counted visually. The minute colonies arising from abortive transduction were counted by examination of 20 or more fields on each of duplicate or triplicate plates 
for calculation of the number of minute colonies per plate. The fields were obtained randomly in some experiments and in others were obtained by counting colonies in adjacent fields across the diameter of the plate twice. Counting of minute colonies by the two methods gave comparable results as did duplicate plates on the same culture. The counts deviated from one another $30-60 \%$ in various tests.

A number of methods of delayed enrichment may be applied in order to obtain macroscopically visible colonies from the minute colonies obtained on minimal medium. Best results were obtained by removing, after incubation for 2 or 3 days at $37^{\circ}$, the entire agar disk from one plate and depositing it in a clean sterile plate to which $0.5 \mathrm{ml}$. distilled water containing $150 \mu \mathrm{g}$ L-histidine had previously been added. A second method, used with temperature sensitive recipients, involved a temperature change after the initial 2- to 3-day period of incubation at $37^{\circ}$. The plates were re-incubated at $24^{\circ}$ for $8-18 \mathrm{hr}$. for the development of the minute colonies into visible clones. Under each of the above conditions the background bacteria (those unaltered with respect to histidine requirement) were also able to proliferate. Microscopic examination showed that neither method achieved quantitative recovery of the minute colonies as visible clones.

The general nature of the minute colonies was determined by picking wellisolated minute colonies with a small platinum loop into a drop of water deposited on a fresh minimal agar plate and streaking into a small sector surrounding the droplet. Eight colonies can be streaked into individual sectors of a single plate. After incubation for 2 days, the streaks were examined microscopically for the presence of minute colonies. The success in detecting minute colonies in individual streaks, for reasons unknown, varies greatly (from $0 \%$ to about $50 \%$ ) in different experiments. The minute colonies in the streaks were also transferred and streaked again in the same matter. In all streaking experiments, care must be taken to avoid disrupting the agar surface as this gives rise to an amount of debris among which it is difficult to detect true minute clones.

Pretreatment of phage suspended in T 2 buffer was performed by utilizing Armour crystalline ribonuclease $(0.5 \mathrm{mg} . / \mathrm{ml}$.) or deoxyribonuclease $(5 \mu \mathrm{g})$. $\mathrm{ml}$. Nutritional Biochemical Company; shown to be very active in inactivating pneumococcus-transforming deoxyribonucleic acid) at $37^{\circ}$ for $30 \mathrm{~min}$. Phage antiserum was prepared according to the procedure of Adams (1959).

\section{RESULTS}

\section{Appearance of minute colonies}

Plate 1, fig. 1, illustrates the large and small colonies obtained as a result of complete transduction under conditions allowing growth of the donor strain (Hartman et al. 1960). These colonies are readily differentiated from the much smaller, minute colonies resulting from abortive transduction (Pl. 1, figs. 2,3). The minute colonies were microscopic in size and accentric in shape. Under optimal plating conditions, they became just barely discernible by the 
naked eye under proper lighting conditions; some were detectable as minute points on the original prints of $\mathrm{Pl}$. 1, fig. 1. When the minute colonies were restreaked on minimal medium agar, not more than one minute colony arose along the streak after incubation. This is in contrast to the behaviour of donor and wild-type colonies found after complete transduction. No confusion in classification resulted from the presence on a single plate, of three types of transductional clones. Restreaking to check on the nature of the minute colonies was performed in $c .30$ instances, including experiments involving intragenic crosses with mutants of the $E, B$ and $D$ loci.

When the minute colonies are considered as approximately circular in outline and the diameter of a number of colonies measured, histograms of the measurements reveal an approximately normal distribution of colony sizes, except for an occasional excess of very small colonies. The comparatively large number of bacteria which constitute the minute colonies after the first $60 \mathrm{hr}$. of incubation under optimal conditions requires that an average of at least five divisions be undergone by each daughter segregating from the presumedly rapidly multiplying organism carrying the transduced fragment (also see Ozeki, 1956). The minute colonies of $E$ mutants were slightly larger and differed morphologically from those of all the other strains examined. The individual bacteria appeared elongate, giving rise to an exceptionally stringy or snaky appearance of the minute colony. $E$ mutants also produced clones of similar morphology on slightly enriched medium (Hartman et al. 1960).

\section{Recessive nature of his mutant genes}

The ability of a combination of the transduced genetic fragment and the host genome to co-operate in allowing minute colony formation on minimal medium will be referred to as an ability for them (or the bacterial strains) to complement one another (complementation). When minute colonies are absent, the two genomes are operationally defined as non-complementary.

With each of over 200 mutants tested, phage prepared on wild-type bacteria has engendered minute colony formation, in addition to large colonies arising from complete transduction. The ratio of minute:large colonies was 8-9:1. This ratio was not appreciably altered by incubation at $24^{\circ}$ or at $15^{\circ}$, although the total number of colonies, due to complete and to abortive transduction, decreased with decreasing temperature. The multisite mutation his-5\%, is completely refractory to complete transduction to wild-type (Hartman et al. 1960). Minute colonies were elicited from his-57 only when phage prepared on cells wild-type for the entire his region were utilized as donors. These minute colonies were, however, much smaller than those normally produced with all of the other his recipients. Thus, his-57 remained as the only strain in which the his mutation did not appear completely 'recessive' to the wild-type allele(s).

\section{Phage transfer of genetic fragments}

The number of abortive transductions was proportional to the multiplicity of phage infection and was decreased in parallel to plaque-forming units when the phage suspension was pretreated with anti-PLT 22 antiserum. The activity 
of the phage in minute colony formation was sedimented with phage particles. Pretreatment of the phage suspension by ribonuclease or deoxyribonuclease had no effect on the numbers of abortive transductions recovered.

The ratio of abortive:complete transductions and the absolute transduction frequencies appeared to be independent of the manner in which phage was grown on wild-type bacteria in multistep lysates. The ratio was the same when the phage was grown at $30^{\circ}$ or at $37^{\circ}$, on minimal medium, on minimal medium supplemented with $\mathrm{L}$-histidinol $(30 \mu \mathrm{g} . / \mathrm{ml}$.), L-histidine $(20 \mu \mathrm{g} . / \mathrm{ml}$.), or spermine (Spermin-phosphate, Hoffman-LaRoche and Co., Basle, Switzerland) (20 $\mu \mathrm{g} . / \mathrm{ml}$.). There was some indication in a few experiments that phage produced on minimal medium (with or without addition of L-histidine) produced a slightly lower ratio of abortive:complete transductional clones than that obtained with phage grown in nutrient broth. This observation was not consistently obtained and variations in the ratio were observed between phage preparations grown in multistep lysates on the same medium. For example, one nutrient broth-grown lysate elicited an exceptional 28:1 ratio and one phage preparation made on minimal medium elicited a 22:1 ratio.

The possibility was considered that abortive transductional clones resulted from the presence of genetic fragments remaining in the recipient cell following recombinational events linked to, but outside of, the mutant locus of the recipient bacterium. This prospect was examined by streaking, on minimal medium, donor-type transductional clones (hisB donors) arising on $\mathbf{E M}+$ histidinol medium from $h i s D$ recipients. No minute colonies were detected out of 200 donor type colonies streaked on minimal medium.

\section{Intergenic tests between his mutants}

The single-site his mutants are divided into seven classes, $A$ to $G$. Some of the mutants are placed on a linkage map through the use of complete transduction tests. The presence of at least seven linearly-arranged and adjacent genes in the histidine region has been indicated. The alleles of a gene represent the members of one of the seven classes (Hartman et al. 1960). Over 5000 combinations of mutants in these different classes have been tested in pairs involving members of each of two genes, for example an $A$ mutant (x) a $B$ mutant, etc. The crosses were examined for the presence or the absence of minute colonies on minimal medium. In almost all of these tests, the presence of minute colonies was detected. Furthermore, the sizes of the minute colonies were the same as those present in parallel tests with wild-type as donor. Genetic complementation was thus generally demonstrable between nonallelic mutants, and with the restoration of an apparently completely wildtype phenotype.

Mutants of class $A$ gave minute colonies in combinations with two phenotypically similar mutants, his-32 and his-107, located between them and gene $B$. The two latter mutants have been given a new letter designation as possibly comprising alleles of a new gene, $H$ (see Fig. 3 and table 3 in Hartman et al. 1960). As indicated below however, the two mutants may actually represent 


\section{Complementation mapping of histidine mutants}

mutations in a second subunit of the $A$ gene. The use of the letter designation $H$ for the two mutants is thus a tentative nomenclature whose verification must await further study. However, it should be noted that both his-32 and his-107 complement all $\boldsymbol{A}$ mutants tested (listed in table 3, Hartman et al. 1960).

Since the formation of minute colonies is not expected to require genetic recombination at the chromosomal level, but merely the functioning of the introduced genetic fragment, it may be predicted that platings on minimal medium of very closely linked non-allelic markers would have a very high ratio of minute: wild type colonies. This was found to be the case. The ratio, which was 8-9:1 when phage grown on wild-type bacteria was used as donor, increased to values as high as $c$. 1000:1 in certain combinations involving mutants of two adjacent genes. However, when the donor type colonies were recovered on the same plate, the ratio of abortive : complete (donor type + wildtype recombinants) was again about $8: 1$.

The sizes of the minute colonies formed in tests involving mutants of two different genes was generally the same as that found when a fragment from a wild-type bacterium was placed in combination with a single mutant. Some exceptions to this generalization occurred, however. Phage prepared on any one of the $\mathrm{F}$ mutants produced smaller minute colonies on hisA-33 recipient than did phages prepared on wild-type bacteria or on cultures of strains mutant at any other gene. As donor, his A-33 again exhibited smaller than customary minute colonies only with $F$ mutants. Similar behaviour was noted with his $B-12$ in combinations with $H$ mutants, his $B-21$ with both $H$ and $A$ mutants, hisB-22 with $H, A$ and $C$ mutants. Correlated with the behaviour of $h i s-22$ in complementation tests was the low concentration detected of imidazoleacetol phosphate ester transaminase, as contrasted with a normal concentration of L-histidinol dehydrogenase (Ames et al. 1960).

A few strains, both as donor and as recipient, give unusually small minute colonies with all mutant strains tested, in addition to the complete absence of minute colonies in tests with allelic mutants. Cell-free extracts of $h i s D, G-63$ which poorly complemented all other mutants, including all $C$ mutants, contained no detectable activity for the four enzyme activities examined (Ames $e t$ al. 1960). Since the interactions leading to decreased minute colony size have not been defined further than the genetic level, we will term the effect a 'position effect'.

The following mutants were found to produce abnormally small minute colonies in combination with mutants of the loci indicated in parentheses: his-B-12 $(H), h i s B-14(H), h i s B-21(H, A)$, hisB-22 (H,A,C), hisB-24 $(H)$, hisB-29 (H) hisH-32 (F, A, B), hisA-33 (F), his-A38 (F), hisA-48 (F), hisF-58 (A), hisD, G-63(E,F,A,H,B,C), hisF-71(E), hisA-80(F), hisA-100 (F), hisE-145 (F), hisC-202 (E,F, A, H, B, D, G), hisG-203 (D and, to a lesser extent, $E, F, A, H, B, C)$ and $h i s D-216(G)$. A further characteristic of the position effect may be noted, namely: it did not skip genes but, rather, was exerted on a continuous chromosome region. Position effects may be much more frequent than indicated since most of the survey was performed without precise measurements of the sizes of the minute colonies produced. 
When two strains, mutant in different genes and each bearing a position effect on the alternate locus in question, were crossed, minute colony formation may be abolished or the colonies decreased in size so as to make the test open to serious question by the unprejudiced observer. For example, hisF-58 elicited abnormally small minute colonies in combinations involving most $\boldsymbol{A}$ mutants. Two $A$ mutants, his $A-33$ and $h i s A-38$, elicited abnormally small minute colonies in combinations involving most F mutants. Crosses of hisF-58 ( $\times$ ) his $A-33$ or hisA-38 produced no discernible minute colonies, forming a non-complementary group. Were we not able to test strain his-58 against a large number of $A$ mutants, a negative complementation result would be scored and a different interpretation placed on the data.

A unique property of $E$ mutants (Hartman et al. 1960) is their ability to bring about the production of larger minute colonies when used as donors than do strains mutant in any other his genes or does the wild-type strain. These minute colonies always have the stringy phenotype of the $E$ mutants. The average diameter of abortive transductional clones formed with hisA-89 recipient were estimated for the following donors (in $\mathrm{mm}$.): $+=0.055$ and 0.054 (duplicate controls), hisE-11 $=0.073$, hisF-6 $=0.052$, hisH $-32=0.039$, hisB-20 $=0.052$, his $C-2=0.053$, and hisD-1 $=0.055$. While the absolute sizes of the minute colonies varied in different experiments, the relative sizes did not change. The minute colonies formed on $E$ recipients were also unusually large, even when phage grown on wild-type bacteria was used. Abortive transductional clones involving his $E, F-135$ were of the $F$ phenotype (i.e. neither stringy nor abnormally large).

\section{Intragenic tests}

When a particular mutant strain was infected with phage prepared on a separate culture of the same strain, it did not yield either wild-type recombinants or minute colonies. The only exception to this finding that we have detected involved infection of $h i s-32$ with phage prepared earlier on his-32 bacteria. Under ideal plating conditions barely perceptible minute colonies were formed. Since his-32 is a 'leaky' mutant, it appears probable that the cell was enabled to produce slightly more effective enzyme activity where two doses of the leaky genes were present together with the rest of the wild-type histidine genes (Hartman \& Hartman, 1957).

Representative alleles from several of the genes were unable to elicit minute colony formation with all other strains tested which were mutant in the same gene as determined by phenotypic characteristics (accumulations and other properties; see Table 2, Hartman et al. 1960) and by location of the site of mutation through recombination analysis. Thus, no minute colonies were obtained from any combination tested involving a large number of $F$ mutants as donors and two $F$ mutants as recipients. In addition, strains hisF-6, F-41, F-42, F-44, F-45, F-58, F-71, F-73, F-76, F-91 and $F-95$ were tested in all possible combinations as donors and as recipients. The same negative result was obtained in tests involving all possible combinations of: (1) $A$ mutants: his-3, $-30,-33,-38,-48,-67,-69,-80,-81$; (2) $H$ mutants: 
his-32, -107; (3) C mutants: his-2, -8, -15, -28, -31, -43, -50, -62, -201, -202, $-207,-209,-210$, and (4) $G$ mutants: his-46, -52, -70, -72, -200, -204, -205. Continued absence of minute colonies within single classes has been noted by testing on spot plates many additional mutants against phages prepared on several mutants from their respective phenotypic classes.

It should be noted that a test for complementation is operationally termed negative when there are absolutely no minute colonies present or, as in a few cases observed, when the number of minute colonies is very very few (less than $0 \cdot 1 \%$ of the expected number when normal ability to complement occurred). The nature of these rare minute colonies has not been determined.

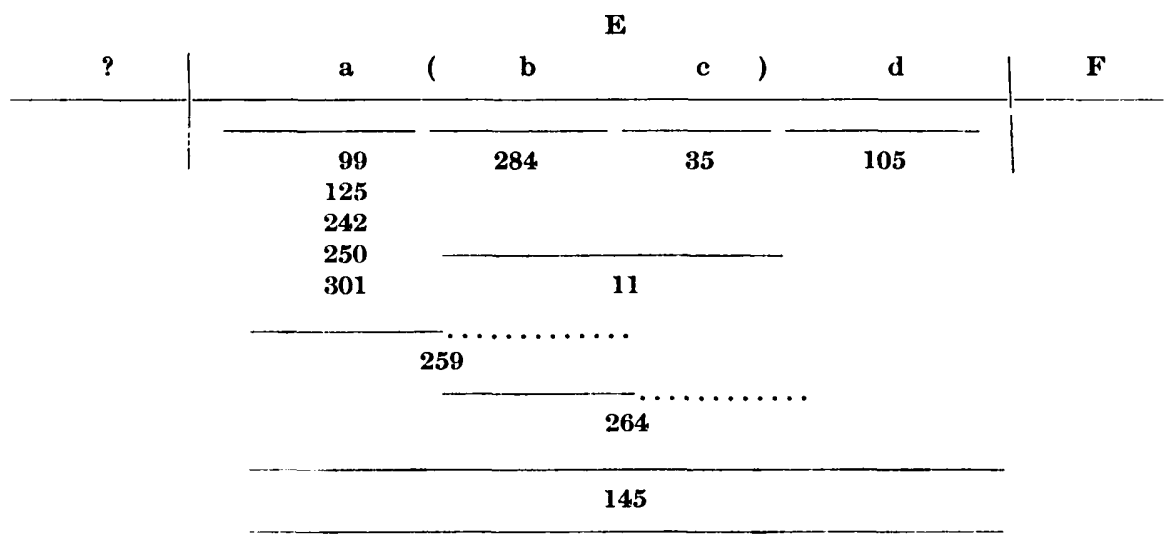

E, F-135

Fig. 1. Schematic representation of complementation behaviour within locus $E$. Numbers are those of the $E$ mutants used in the tests. Solid lines indicate absence of minute colony formation in reciprocal tests. Dotted lines indicate weak complementation (minute colony size smaller than obtained in combination with wild-type). Comparisons are to be made in vertical columns. For example, mutant his-264 failed to complement his-284, $-11,145,-135$, complemented his-35 only weakly, and fully complemented all other $E$ mutants listed.

Unexpected complementation occurred between mutant alleles within genes $E, B$ and $D$. Minute colony formation occurred in combinations involving some pairs of $\boldsymbol{E}$ mutants but not between certain other pairs. Where complementation occurred, the minute colonies exhibited excessive size and 'snaky' appearance characteristic of intergenic crosses involving $E$ mutants. Figure 1 schematically represents the complementation reactions noted for $E$ mutants. It should be stressed that Fig. 1 is a 'complementation map' (see Giles, 1958); its relation to genetic mapping by linkage studies remains to be determined. The mutants are divided into a number of classes involving 4 basic groups, $a$ to $d$. Mutant his-99, an $E a$ mutant, was able to complement his-284, $-35,-11,-105$, and -264 , but unable to complement itself or his-125, $-242,-250,-301,-259,-145$, or -135 . His-11 is termed an Ebc mutant since it failed to complement two mutants (hisEb-284 and hisEc-35) which complemented one another. Mutant his-145 failed to complement any $E$ mutant ('hisEabcd-145'), while hisEabcd, F-135 complemented no $E$ or $F$ mutant. 
Since his-135 gave recombinants with all $E$ mutants currently available, one cannot be sure that the inability to complement is due to 'position effect' or to actual mutational involvement of the $E$ gene itself. For example, in his-135 the mutational site may extend from gene $\boldsymbol{F}$ into gene $\boldsymbol{E}$ over a portion of the map corresponding to a site in which a single mutant would give the abcd complementation pattern. Mutant his-259 appeared to produce smaller than normal minute colonies with his-284 and, especially, his-11 (dotted line in Fig. 1); however, high-back mutation frequency in his-259 and -284 prevented precise analysis.

\begin{tabular}{|c|c|c|c|c|}
\hline \multirow[t]{2}{*}{$\mathbf{H}$} & \multicolumn{4}{|c|}{ B } \\
\hline & $\mathbf{a}$ & $\mathbf{b}$ & c & $\mathbf{d}$ \\
\hline & 20 & B] & & O \\
\hline & 79 & 206 & 59 & 47 \\
\hline & & & 116 & 143 \\
\hline & & & 136 & 213 \\
\hline & & & & 217 \\
\hline & & & & 229 \\
\hline & & & & 238 \\
\hline & & 53 & & 257 \\
\hline & & & & \\
\hline & 12 & & & \\
\hline & 14 & & & \\
\hline & & & 234 & \\
\hline & & & & 65 \\
\hline & & & & \\
\hline & & & & \\
\hline & & & & \\
\hline & & & & \\
\hline & & & & \\
\hline & & & & \\
\hline & & & & \\
\hline & & & & \\
\hline & & & & \\
\hline
\end{tabular}

Fig. 2. Schematic representation of complementation behaviour within locus $B$. Diagrammed as for $E$ locus (see legend to Fig. 1).

Complementation reactions between alleles of the $B$ genes similarly indicated the presence of 4 basic groups ( $a$ to $d$ in Fig. 2). A larger number of mutants available for analysis and mutants which showed over-lapping effects in the complementation reactions allow further generalizations to be made. It would appear that there are probably only a very limited number of basic complementation classes. Most of the mutants either fell into a single class, 
complementing the other three classes, or were unable to complement all four basic classes. Some mutants which did not complement one class produced very weak responses with one or more of the remaining classes. The 'weak response' was due to a uniformly small size of all minute colonies formed, rather than to decreased numbers of minute colonies. The existence of mutants whose complementation reactions overlapped two or three classes either completely (e.g. hisBcd-102, hisBbcd-234) or by decrease in minute colony size (e.g. hisBa-12) permits only one linear arrangement of classes with effects on a continuous gene region. Extensive two-point tests indicated the members of each basic complementation class as forming a very closely-knit set of mutations within one portion of the gene (Hartman et al. 1960; and unpublished). Each of the mutants was separable by recombination in complete transduction from each of the other mutants with the following two exceptions (Hartman et al. 1960): (1) The pairs, his-40 and -47; his-12 and -24; and his-53 and -206. The two members of the pair, in each case, gave qualitatively identical patterns of complementation although his-5.3 and -206 differed quantitatively (see Fig. 2). (2) Mutant his-22 was unable to elicit wild-type recombinants by complete transduction with any $B$ mutant.

Alleles his-12 and - 24 were transduced into a culture of his-1 derived from a single colony. Two clones from each of the donors were isolated and found to exhibit the specific intragenic and intergenic complementation pattern typical of $h i s-12$ and $h i s-24$. The complementation behaviour was thus due to the genetic constitution of the his region itself, not to unlinked or loosely linked modifiers.

Mutations of gene $D$ assorted into one of three categories by abortive transduction tests: (1) $D a$ mutants, which were able to complement $D b$ mutants but neither $D a$ nor $D a b$ mutants ; (2) $D b$ mutants, which complemented $D a$ mutants but neither $D b$ mutants nor Dab mutants; (3) Dab mutants, unable to complement $D a, D b$ or $D a b$ mutants. Contrary to the behaviour in the other two genes which showed intragenic complementation, many of the $D$ mutants appear to be of the non-complementing ( $D a b)$ type. Only six $D a$ mutants have been discerned (his-10,-23, -25,-112, -126, and -208); the complementation observed with his $D a-25$ may be partially temperature sensitive. Many more mutants were classified into the $D b$ category: his-1, $-36,-37,-39,-49,-66,-68,-77,-82,-83,-84,-90,-92,-108,-111,-113,-130$, $-141,-214,-223,-236,-237,-239,-244,-245,-248$, and -254 .

Analysis of $D$ mutants by recombination techniques supplied a more reliable mapping of the $D$ gene than that obtained for any of the other his genes (Fig. 4, Hartman et al. 1960). Mutants determined as of the Da type by abortive transduction (marked with $\times$ 's in the figure) mapped on the 'left' half of the gene $D$; those determined as $D b$ mutants mapped within the 'right' half of gene $D$. Dab mutants did not map in the centre of the gene but, rather, appeared to be scattered through the 'right' half of the gene. All four of the strains which exhibited recombination with the multisite mutant his-152, and therefore presumed to be located in the far 'right' half of the $D$ gene (Fig. 4 of Hartman et al. 1960), were Dab mutants. 


\section{Complementation behaviour of multisite mutants}

The extent of the region structurally altered in multisite mutants is defined first by recombinational analysis through the use of complete transduction. One may also examine the multisite mutants by the abortive transduction test for genetic complementation. The results reveal some striking concordances and a few discrepancies between the two methods of analysis (refer to Fig. 3 of Hartman et al. 1960).

(1) Mutant his-41 failed to give recombinants with some $F$ mutants and failed to complement all of the $\boldsymbol{F}$ mutants but complemented mutants of all other genes. Similarly, his-55 failed to give recombinants with some $F$ mutants and some A mutants and failed to complement alleles of genes $E$ or $A$ but complemented all other genes. Mutant his-152 produced no recombinants in combination with any $A, H, B$ or $C$ mutant and with only a few $F$ and $D$ mutants and failed to complement mutant alleles of these genes but complemented genes $E$ and $G$. The minute colonies in combinations of his-152 and $E$ mutants were large and 'stringy', typical of those obtained in other tests involving $E$ mutants. These tests are thus consistent with predicted theory.

(2) Mutant his F-135 failed to recombine with several $F$ mutants but recombined with all $E$ mutants; it complemented no $E$ or $\boldsymbol{F}$ mutant. However, since there was only a limited number of $\boldsymbol{E}$ mutants available for testing it is assumed that the aberration in his-135 actually extended into the $E$ gene (as mentioned above). A similar assumption would explain the behaviour of stable mutant his-134 which failed to complement $A$ and $H$ mutants and gave recombinants with both $\boldsymbol{H}$ mutants and all but one $\boldsymbol{A}$ mutant. Recombination tests showed his-134 to be located between $h i s-A 3$, and the closest member of the adjacent gene hisH-10\%.

(3) Mutants his-22 and -32 did not produce wild-type recombinants with one another, yet they elicited minute colony formation following transduction and plating on minimal medium. Complementation was also observed between $h$ is $B-22$ and $h i s H-107$, further indicating a localization of genetic damage to the $B$ gene in $h i s-22$. A similar case was found with $h i s D, G-63$; this mutant failed to recombine with any $D, G$ and with three $C$ mutants (his-62, -202, -209). It produced no minute colonies with $D$ or $G$ mutants yet minute colonies were detected in combinations involving all $C$ mutants, including the three strains mentioned above. These results indicate localization of the actual genetic damage to the $D$ and $G$ genes and an inhibition of recombination outside of the mutated region.

(4) Mutant his-57 was unable to form minute colonies, either as donor or as recipient, in combination with any of the his mutants. Phage grown on wildtype bacteria elicited only very small minute colonies on his-57 recipient. In contrast, when used as donor his-57 formed wild-type recombinants with all $E, F, A, H$, and most $B$ mutants (Hartman et al. 1960). 


\section{DISCUSSION}

The present work indicates that a linear region of the Salmonella typhimurium chromosome is the seat of all genetic information required for the synthesis of L-histidine by the primary metabolic pathway (outlined in fig. 3, Hartman et al. 1960). This region is divided into smaller non-overlapping units or genes. Each gene is conceived as having a discrete function, probably a role in the determination of the structure of a single enzyme or protein molecule. Some of the genes also appear to be functionally unitary in the genetic sense, but others are composed of 2, or of 4, functional subunits. Figure 4 in Hartman et al. (1960) shows a map of the hisD gene. It appears to consist of two functional regions, $D a$ and $D b$. Cell-free extracts of hisDa-10 and hisDb-1 bacteria lack detectable histidinol dehydrogenase activity, as do mixtures of these two extracts (Ames et al. 1960).

Demerec \& Hartman (1959) reviewed additional instances of the presence of subgenic complementation units in micro-organisms, and Carlson (1959) discussed the structure of genes in Drosophila. An attractive hypothesis would relate the functional subunits of the genes to the synthesis of individual polypeptide chains or protein subunits of particular enzymes. This view is similar to Benzer's hypothesis concerning the possible functions of similar units ('cistrons') in bacteriophage (Benzer, 1955). Non-complementing sites (e.g. $D a b$ ) may be considered either as involving some residue critical for binding enzyme subunits together or as exerting a 'position effect' on neighbouring complementation units when in an altered configuration. In either event, the presence of non-complementing mutants allows some decision, on genetical grounds, about the definition of the limits of the gene locus. The existence of non-complementing mutants in bacteria and Neurospora has excluded these systems from Benzer's definition of the 'cistron'; consequently the term 'complementation unit' has been coined for the basic functional unit (Demerec \& Hartman, 1959).

The formation of unique antigens in hybrid bacteria (reviewed by Hartman \& Goodgal, 1959) and heterozygous organisms (e.g. Chovnick \& Fox, 1953) may be achieved by intragenic complementation as well as by intergenic complementation; the latter possibility was pointed out previously (Beadle \& Coonradt, 1944).

Intragenic complementation also provides an excellent means whereby a population of diploid organisms may maintain a ready supply of two phenotypes, each of which has a selective advantage under one of two alternative environmental conditions; because of close linkage such an arrangement would resist disruption by recombination. Furthermore, the splitting of enzymes into protein subunits by specific small molecules (e.g. Frieden, 1958) might allow the release, at a critical time, of polypeptide units with a high degree of specificity, possibly requisite for the repression or activation of specific genes (compare Markert, 1958) or enzyme-forming centres.

The complementation units, like the genes, appear to be complex linear lengths of genome, divisible both by mutation and by recombination. If the 
penicillin procedure is effective in the isolation of a true sample of available mutations, our data imply that histidine mutations are not equally divided between the complementation units of single genes (e.g. compare frequencies of $D a$ and $D b$ mutants). This might be due to: $(a)$ a weak or absent histidine phenotype engendered by mutation at many sites of a particular complementation unit (witness that most mutants of subunit $B c$ are leaky; see Gots, Coon \& Hild, 1959); (b) a non-random spatial distribution of mutations themselves; (c) genetic inertness of portions of certain subunits; $(d)$ a true inequality of genetic sizes.

The data are consistent with the ideas that the leaky nature of some alleles is determined both by the positions (sites) at which mutation has occurred, and in the nature of the structural changes themselves. A single site may exist in at least three forms (see Demerec \& Hartman, 1959). For example, of the set of mutants which produce no wild-type recombinants in reciprocal crosses, his-2 is not leaky but $h i s-211$ is leaky and temperature sensitive. Furthermore, some leaky mutants respond to chemical mutagens with presumed specificity of action as do other non-leaky mutants localized elsewhere in the same gene or complementation unit (Kirchner, personal communication).

It appears that some specific mutations frequently exert a position effect, i.e. inhibit the expression of nearby gene(s). As found in most cases in higher organisms, this inhibition is only partial (see examples and discussions in Muller \& Prokofyeva, 1935; Lewis, 1950). A similar effect is often noted at the intragenic level when complementation units are present, suggesting that the two phenomena may be related. When tested with phage grown on wild-type bacteria, most of the mutant alleles appear to behave as complete recessives. This would argue against any hypothesis suggesting the formation, by the mutant allele, of abnormal enzyme or other substance inhibitory to the functioning of normal enzyme. The position effects are also specific as to the gene or genes whose expression is affected. The position effects are exerted on continuous chromosomal regions. A behaviour of this type might be expected if isoalleles were present in our various strains for a gene or genes different from that in which the primary mutation occurred (i.e. that other 'wild-type' genes often carry alterations with but minor effects on the phenotype). Here one cannot adequately account for the unique distributions of the position effects nor the presence, in a particular stock, of a number of such presumed isoalleles. A third alternative, namely, the assumption of the presence of 'modifiers' unlinked with the his genes, is excluded by the persistence of position effects in two mutants transduced into otherwise isogenic stocks. The interpretation remaining would endow the altered allele itself with a direct effect on the functioning of nearby complementation units or genes which, themselves, were structurally unaltered. This effect might occur at the chromosomal level, or, if the his region serves to produce a giant multi-enzyme forming template, at the level of enzyme formation.

The correspondence of gene function with genetic complementation allows rapid placement into gene loci on a chromosomal map of phenotypically 


\section{Complementation mapping of histidine mutants}

similar mutants on the basis of complementation alone. Further, the correspondence of the intragenic linkage maps of mutant sites in the his $\boldsymbol{D}$ gene (Fig. 5, Hartman et al. 1960) and the intragenic ordering of complementation units by abortive transduction tests (Figs. 2, 3) indicate that the latter method may also have some value in the intragenic placement of mutational sites for complementing mutants (see Giles, 1958). Indeed, we now 'place' mutants into very small regions of the linkage map by just this method; in all cases where unequivocal results are obtained in the complementation tests, the mutant has satisfied all other criteria (linkage relations and phenotype) predicted for it.

The abortive transduction test gives a very sensitive measure of enzyme production. For example, hisB-22, which weakly complements $h i s-C$ mutants, contains under optimal conditions of growth only a few per cent of the normal concentration of the enzyme controlled by the $C$ gene (imidazoleacetol phosphate ester transaminase; Ames et al. 1960).

This investigation was supported by a research grant, E-1650, from the National Institute of Allergy and Infectious Diseases, Public Health Service, U.S.A.

\section{REFERENCES}

Adams, M. H. (1959). Bacteriophages. New York: Interscience Publishers, Inc.

Ames, B. N., Garry, B. \& Herzenrerg, L. (1960). The genetic control of the enzymes of histidine biosynthesis in Salmonella typhimurium. J. gen. Microbiol. $22,369$.

Beadle, G. W. \& Coonradt, V. L. (1944). Heterocaryosis in Neurospora crassa. Genetics, 29, 291.

BEnzer, S. (1955). Fine structure of a genetic region in bacteriophage. Proc. nat. Acad. Sci., Wash. 41, 344.

Carlson, E. A. (1959). Comparative genetics of complex loci. Quart. Rev. Biol. 34, 33.

Chovick, A. \& Fox, A. S. (1953). Immunogenetic studies of pseudoallelism in Drosophila melanogaster. I. Antigenic effects of the lozenge pseudoalleles. Proc. nat. Acad. Sci., Wash. 39, 1035.

Demerec, M. \& Hartman, P. E. (1959). Complex loci in microorganisms. Annu. Rev. Microbiol. 13, 377.

Demerec, M. \& OzekI, H. (1959). Tests for allelism among auxotrophs of Salmonella typhimurium. Genetics, 44, 269.

Frieden, C. (1958). The dissociation of glutamic dehydrogenase by reduced diphosphopyridine nucleotide (DPNH). Biochim. biophys. Acta, 27, 431.

Gines, N. H. (1958). Mutations at specific loci in Neurospora. Proc. 10th int. Congr. Genetics, Montreal, 1, 261.

Gots, J. S., Coon, R. G. \& HiLd, D. H. (1959). Cistronic control of biosynthetic leakage in purine auxotrophs of Salmonella typhimurium. Bact. Proc. p. 35.

Hartman, P. E. (1957). Transduction: a comparative review. In $A$ Symposium on The Chemical Basis of Heredity, ed. W. D. McElroy \& B. Glass, pp. 408-62. Baltimore: The Johns Hopkins Press.

Hartman, P. E. \& Goodgal, S. H. (1959). Bacterial genetics (with particular reference to genetic transfer). Annu. Rev. Microbiol. 13, 465.

Hartman, P. E. \& Hartman, Z. (1957). Histidine-requiring mutants of Salmonella typhimurium: Complete and abortive transduction. Heredity, 11, 286. 
Hartman, P. E., Loper, J. C. \& Šrman, D. (1960). Fine structure mapping by complete transduction between histidine-requiring Salmonella mutants. J.gen. Microbiol. 22, 323.

LeDERBERG, J. (1956). Linear inheritance in transductional clones. Genetics, 41, 845. Lewis, E. B. (1950). The phenomenon of position effect. Advanc. Genet. 3, 73.

Markert, C. L. (1958). Chemical concepts of cellular differentiation. In Chemical Basis of Development, ed. W. D. McElroy \& B. Glass, pp. 3-16. Baltimore: The Johns Hopkins Press.

Muller, H. J. \& Prokofyeva, A. A. (1935). The individual gene in relation to the chromomere and the chromosome. Proc. nat. Acad. Sci., Wash. 21, 16.

OzEKI, H. (1956). Abortive transduction in purine-requiring mutants of Salmonella typhimurium. Genetic Studies with Bacteria. Publ. Carneg. Instn, no. 612, p. 97.

Stocker, B. A. D. (1956). Abortive transduction of motility in Salmonella. J. gen. Microbiol. 15, 575.

Stocker, B. A. D., Zinder, N. D. \& Lederberg, J. (1953). Transduction of flagellar characteristics in Salmonella. J. gen. Microbiol. 9, 410.

\section{EXPLANATION OF PLATE}

Fig. 1. Petri dish containing Salmonella typhimurium mutant his-12 recipient infected with phage grown on his-56. Plated on minimal (E) medium containing adenine, methionine, thiamine and pantothenate and incubated for $72 \mathrm{hr}$. at $37^{\circ}$. The large colonies are clones of wild-type recombinants ; the small colonies represent donor-type recombinants.

Fig. 2. Portion of a Petri dish, showing his-11 recipient infected with phage grown on his-56. Plating conditions as in fig. 1. Part of a large colony derived from a wild-type recombinant is on the left with a small colony of donor-type bacteria (his-56) adjacent to it. Arrows indicate positions of minute colonies resulting from abortive transduction. Enlarged $c . \times 25$.

Fig. 3. Enlargement of a portion of a Petri dish which has been spread with bacteria of a $h i s B$ recipient infected with phage grown on wild-type bacteria. Incubated for $48 \mathrm{hr}$. at $37^{\circ}$ on minimal medium. The edge of a wild-type colony resulting from complete transduction is at the bottom of the photograph. A minute colony is designated by an arrow. The 'background' bacteria which have not received any hisB' gene have failed to divide or have divided only once or twice; they appear as beaded chains, scattered on the surface of the agar. Enlarged about $\times 250$. 
Journal of General Microbiology, Vol. 22, No. 2

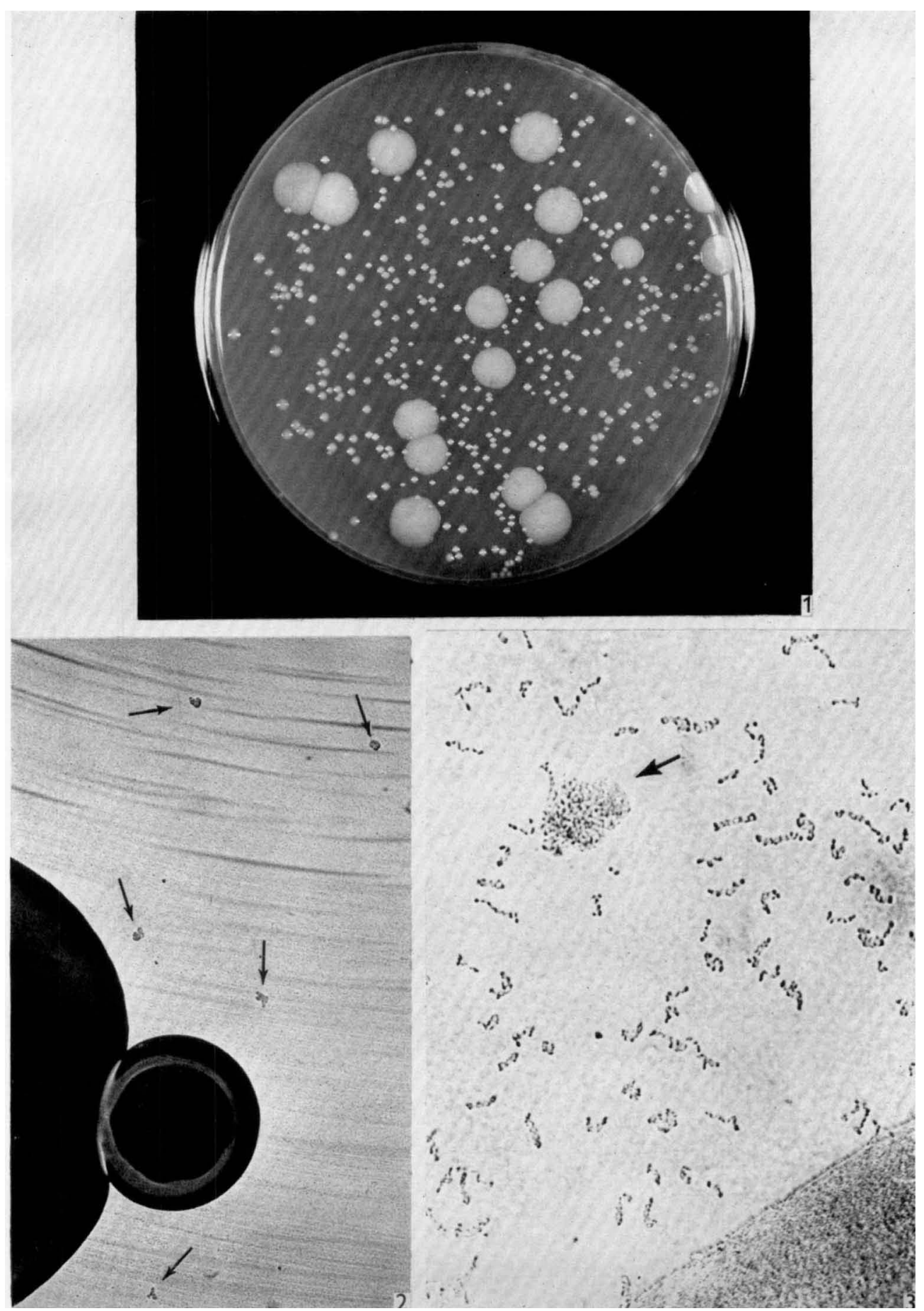

P. H. HARTMAN, Z. IIARTMAN \& 1). S̈ERMAN (COMPLENENTATON MAPPING OF HISTIDINE MUTANTS. PIATE 1 